\title{
Vaterschaftstests in der Arztpraxis
}

\author{
S. Braga
}

Zunehmend wird die Ärzteschaft von Laboratorien mit Werbung zu Vaterschaftstests versehen. Einerseits ist die Reaktion auf die Werbung eine klare Ablehnung, andererseits besteht eine gewisse Verunsicherung. Dazu eine persönliche Meinung, in welche die Gedanken aus Diskussionen mit mehreren Personen aus verschiedenen Berufsrichtungen eingeflossen sind. Es wird der grundsächlichen Frage nachgegangen: Haben wir als Ärztinnen und Ärzte im Kontext von Vaterschaftsabklärungen überhaupt eine Aufgabe? Juristische Aspekte werden bewusst ausgelassen. Entscheidungen, die unser ärztliches Verhalten betreffen, beruhen in erster Linie auf einer ethischen Reflexion, nicht auf gesetzlichen Vorgaben.

Korrespondenz:

Dr. med. Suzanna Braga

Merzenacker 8

CH-3006 Bern
Vaterschaftstests sind offensichtlich «en vogue»! Ungefähr ein Jahr ist es her, seit sich die Apotheker mit dem Thema befassen mussten, in regelmässigen Abständen flattern Angebote und Informationen von verschiedenen Privatlabors in die Arztpraxen. In der letzten Woche sind wir einmal mehr durch einen offenbar weitgestreuten Versand dazu aufgefordert worden, Vaterschaftstests anzubieten. Im Internet haben sie sich schon längst etabliert. Die neuste Idee kommt von Gentest.ch GmbH, Schlossgasse 9, 8003 Zürich. Weiträumig ist die Ärzteschaft angefragt worden, sich als Zulieferantin für Vaterschaftstests zur Verfügung zu stellen und sich auf die Ärzteliste aufnehmen zu lassen (weitere Informationen: www.gentest.ch). Etwas über 200 Kolleginnen und Kollegen haben sich einspannen lassen.

\section{DNA-Vaterschaftstests sind sehr zuverlässig, aber ...}

Zugegeben, molekulargenetische Untersuchungen liefern die zuverlässigsten Resultate hinsichtlich der Abstammung einer Person, sofern Methodik und Interpretation nach allen Regeln der Kunst durchgeführt werden. Die Asservierung von Untersuchungsmaterial ist an sich einfach. Aber die Identitätsüberprüfung kann eine Falle sein, denn wer garantiert dafür, dass keine Fälschung der Dokumente vorliegt? Eine gesetzliche Regelung ist im Bundesgesetz zur genetischen Untersuchung am Menschen vorgesehen. Dieses ist nach jahrelanger Vorarbeit erst in der Parlamentarischen Kommission. Es kann also noch lange dauern, bis es in Kraft treten wird.

\section{Was ist gutes ärztliches Handeln (good medical practice)?}

Doch es sind nicht in erster Linie die juristischen Aspekte, die mich zu diesem Artikel bewegen, sondern ganz grundsätzliche ärztliche Fragen. Fragen, die sich darauf beziehen, wie wir unseren Beruf verstehen und ausüben wollen. Haben wir als Ärztinnen und Ärzte im Kontext von Vaterschaftsabklärungen überhaupt eine Aufgabe? Gibt es eine medizinische Indikation für eine Vaterschaftsuntersuchung? Ergeben sich aus dem Resultat der Untersuchung allenfalls therapeutische Massnahmen? Ich denke, die Antwort auf diese Fragen ist, abgesehen von einigen wenigen Ausnahmen, klar ein Nein.

Sicher gibt es Patienten oder Patientinnen, die wir schon einige Zeit betreuen, die uns auf das Thema der Vaterschaftsabklärung ansprechen. Hin und wieder taucht die Frage nach der Vaterschaft im Zusammenhang mit psychosomatischen Symptomen oder im Kontext genetischer Fragen auf. In der ärztlichen Beziehung zur Person, die einen Vaterschaftstest wünscht oder in Erwägung zieht, ergibt sich die Möglichkeit zu klären, was hinter dem Anliegen steht und welches Ziel damit erreicht werden soll. Die allfälligen Konsequenzen für alle Betroffenen, auch für das Kind und die Beziehung zu ihm, können diskutiert, gemeinsam nach Lösungen gesucht, die notwendigen Schritte eingeleitet und allenfalls an die richtigen Stellen verwiesen werden.

\section{Reflexion mit Augenmerk auf den Kontext}

Der Wunsch nach einer Vaterschaftsabklärung findet immer in einem besonderen Kontext statt. Sie hat immer eine Vorgeschichte und ein Nachspiel. Im ganzen Verlauf vom ersten In-ZweifelZiehen einer Vaterschaft bis zur Entscheidung, eine Abklärung in Erwägung zu ziehen, treten meist viele Belastungen und Verletzungen auf. Der Bedeutung, welche die Untersuchung und deren Resultat für das Kind hat, wird in der Regel kaum Beachtung geschenkt. Wollen wir im Bewusstsein dieser Tatsachen wirklich als Lieferanten für das Untersuchungsmaterial zur Verfügung stehen? Wie steht es um Einhaltung des 
Versprechens «primum nihil nocere»? Wie ist es möglich, diesem nachzuleben, wenn wir lediglich die Identität der zu Untersuchenden feststellen und dann das Untersuchungsmaterial entnehmen?

Jede Diagnostik setzt eine Indikation voraus und soll Basis zur Therapie oder zu therapeutischen Massnahmen sein. Das griechische Wort, von dem Therapie abgeleitet ist, heisst in seiner ursprünglichen Bedeutung «einen Dienst erweisen». Wem wollen wir einen Dienst erweisen? Wer genau ist oder sind die Partner im Kontrakt der Arzt-Patienten-Beziehung? Eine ethische Haltung vertreten heisst, sie umsetzen und sie leben. Diese Umsetzung findet immer innerhalb einer Beziehung statt. Dies geht auch aus der eben in der Schweizerischen Ärztezeitung veröffentlichten Charta zur ärztlichen Berufsethik [1] deutlich hervor. Lesen Sie die Charta. Bleiben danach noch Fragen in bezug auf ärztliches Verhalten im Bezug zu Vaterschaftstests offen? Die Entscheidung betreffend unser Verhalten beruht in erster Linie auf einer ethischen Reflexion, nicht auf gesetzlichen Vorgaben.

\section{Literatur}

1 Charta zur ärztlichen Berufsethik. Schweiz Ärztezeitung 2003;84(45):2347-9.

\section{Gegendarstellung zu «Cavete» (SÄZ 2003;84[46]:2411)}

1. H+ schliesst zu Lasten der FMH keine Verträge mit Spitälern bezüglich Dignitätsdaten ab.

2. H+ hat einen Mustervertrag erarbeitet, welcher von den Spitälern unter Zustimmung aller Ärzte für die Einforderung der notwendigen Dignitätsdaten bei der FMH eingereicht werden kann.

3. Das Generalsekretariat der FMH hat beim vorliegenden Mustervertrag mitgearbeitet und somit Kenntnis davon.

4. Leider hat die FMH die gemeinsame Publikation des Mustervertrags abgelehnt, jedoch zugesichert, dass Anträge der Spitäler geprüft werden.
Alle Spitäler benötigen die Dignitätsdaten ihrer Ärzte vor dem 1. Januar 2004, um die Abrechnung über TARMED und in vielen Fällen die Honorarzahlungen an die Ärzte realisieren zu können.

H+ Die Spitäler der Schweiz Dr. Uma Grob, Geschäftsführerin Bernhard Wegmüller, Stv. Geschäftsführer 